Rhode Island College

Digital Commons @ RIC

Master's Theses, Dissertations, Graduate

Master's Theses, Dissertations, Graduate

Research and Major Papers Overview

Research and Major Papers

Spring 2017

\title{
Concussion: Improving Nurses' Knowledge in the Emergency Department
}

Danielle Marsden

Rhode Island College

Follow this and additional works at: https://digitalcommons.ric.edu/etd

Part of the Nursing Commons

\section{Recommended Citation}

Marsden, Danielle, "Concussion: Improving Nurses' Knowledge in the Emergency Department" (2017).

Master's Theses, Dissertations, Graduate Research and Major Papers Overview. 192.

https://digitalcommons.ric.edu/etd/192

This Major Paper is brought to you for free and open access by the Master's Theses, Dissertations, Graduate Research and Major Papers at Digital Commons @ RIC. It has been accepted for inclusion in Master's Theses, Dissertations, Graduate Research and Major Papers Overview by an authorized administrator of Digital Commons @ RIC. For more information, please contact digitalcommons@ric.edu. 
CONCUSSION: IMPROVING NURSES’ KNOWLEDGE IN THE EMERGENCY DEPARMENT

\author{
by
}

Danielle Marsden

A Major Paper Submitted in Partial Fulfillment

of the Requirements for the Degree of

Master of Science in Nursing

in

The School of Nursing

Rhode Island College

2017 


\begin{abstract}
Patients presenting to the emergency department (ED) after trauma from a fall, motor vehicle collision, or assault frequently develop a mild traumatic brain injury (MTBI) also called concussion. There is no specific treatment for concussion, therefore, the focus shifts to managing symptoms. Countless EDs experience overcrowding leading to time and resource constraints, therefore nursing discharge education is brief and may only review symptoms which require immediate medical attention. This ED discharge practice leaves the patient on their own to manage symptoms and if the patient is unaware, they may perform activities that exacerbate their condition. The purpose of this project was to evaluate the impact of an educational program on ED nurses' knowledge of concussion/MTBI and the current Centers for Disease Control and Prevention (CDC) recommended education for patients and families discharged home.
\end{abstract}

The study took place at a large level I trauma center, using a nonprobability convenience sample of ED nurses. A pre-intervention post-test design was used to measure nurses' knowledge regarding concussion. The intervention consisted of a poster board and informational packet that provided nurses with evidence-based information about concussion and discharge instructions. Fifty-one nurses (28\%) completed the pretest with a mean score of $61.96 \%$ and total scores ranged from $30 \%$ to $90 \%$. Twentyeight nurses (15\%) completed the post-test with a mean score of $78.2 \%$ and a range of $40 \%$ to $100 \%$. There was an overall improvement in the total scores by $16.24 \%$. These findings suggest that an evidence-based educational intervention increases ED nurses' knowledge regarding concussion and appropriate discharge education for concussion patients. 


\section{Table of Contents}

Background/Statement of the problem ..................................... 1

Literature Review ........................................................ 4

Theoretical Framework .................................................... 21

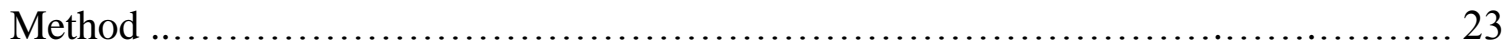

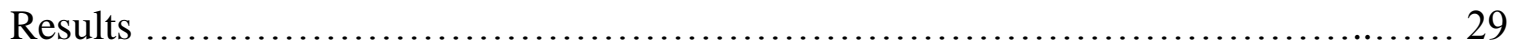

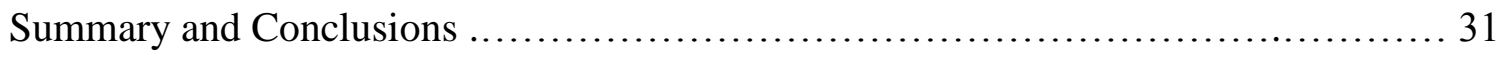

Recommendations and Implications for Advanced Nursing Practice ................. 35

References ............................................................ 37

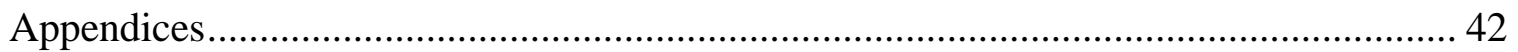


Concussion: Improving Nurses' Knowledge in the Emergency Department

\section{Background/Statement of the Problem}

According to the Centers for Disease Control and Prevention (CDC) in 2010, 2.5

million emergency department (ED) visits, hospitalizations, or deaths were associated with traumatic brain injury (TBI) and from 2001 to 2010, ED visits related to TBI increased by $70 \%$. The most common form of TBI is mild TBI (MTBI), also known as concussion, which accounts for $75 \%$ of those injured (Bergman \& Bay, 2010). “A concussion is a type of TBI caused by a bump, blow, or jolt to the head or by a hit to the body that causes the head and brain to move rapidly back and forth. This sudden movement can cause the brain to bounce around or twist in the skull, stretching and damaging the brain cells and creating chemical changes in the brain" (Centers for Disease Control and Prevention, 2015). Although often considered mild, these injuries can lead to significant physical, cognitive, and emotional impairment.

In the late 1990's, the dangers of concussion became infamous when several former National Football League players committed suicide. They all donated their brains to science and were diagnosed with chronic traumatic encephalopathy (CTE) postmortem. This began discussions about cognitive and mood disorders numerous professional athletes were experiencing and many filed a class action lawsuit against the National Football League. In April 2015, the lawsuit resulted in a \$1 billion award to athletes who have suffered from concussions. To date, 87 football players out of the 91 players who have donated their brains after death have tested positive for CTE (Mapstone, 2016). Currently there is no diagnostic tool for CTE, but the symptoms 
include anxiety, depression, rage, confusion, impaired judgement, memory loss, and ultimately dementia (Mapstone, 2016).

Up to $30 \%$ of patients discharged with a diagnosis of concussion have symptoms at three months and $15 \%$ are symptomatic one-year post head injury (Haydel, 2012). Prolonged symptoms often lead to missed work and productivity, as well as increased healthcare cost. Post-concussion syndrome (PCS) defined by The Diagnostic and Statistical Manual of Mental Disorders (DSM-IV) is "a clinical state where three or more symptoms of concussion persist for more than three months after a head injury" (Sheedy, Geffen, Donnelly, \& Faux, 2006, p. 757). According to Haydel (2012), TBI exceeds \$60 billion annually in the United States including direct as well as indirect costs. The impact of early interventions on PCS can lead to decreased morbidity and healthcare cost (Jagoda et al., 2008).

Many individuals come to the hospital following head trauma, the initial focus in the ED is on ruling out more serious injury including cervical neck fracture or brain hemorrhage, which is appropriate, but once the patient is cleared from requiring cervical spine stabilization they are often discharged directly from the ED. There is no specific treatment for concussion, therefore focus shifts to managing symptoms. Countless EDs experience overcrowding leading to time and resource constraints, therefore nursing discharge education is brief and may only review symptoms which require immediate medical attention. This ED discharge practice leaves the patient on their own to manage symptoms and if the patient is unaware, they may perform activities that exacerbate their condition. Often times these patients return to the ED for common concussion symptoms that could have been managed at home if adequate education was provided at discharge 
by nursing staff (Ganti et al., 2015). Many patients have a poor understanding of concussion yet there is a wide variation in concussion education and recommended follow up instructions (Haydel, 2012).

The CDC's HEADS UP campaign (2015) recommends using the Acute Concussion Evaluation (ACE) care plan which has evidence-based discharge instructions that can be used by nurses discharging concussions patients. Educating ED nurses about concussion is crucial because ED nurses play a key role in helping to improve patient health outcomes through patient teaching (Atzema et al., 2013). The purpose of this project is to increase ED nurses' knowledge of concussion/MTBI and the current CDC recommended education for patients and families discharged home. 


\section{Literature Review}

A literature search was conducted using the following databases; PubMed, CINHAL and Cochrane Library, as well as bibliographic reference list searches of relevant articles. Search terms included 'concussion education', ‘emergency department', 'emergency department education', as well as 'concussion' combined with 'treatment', 'guidelines', 'management' and 'prognosis' using the Boolean operator 'AND'. Additional information was obtained from government educational websites such as the Centers for Disease Control and Prevention, HEADS UP Campaign. The articles ranged from the years 1996 to 2016.

\section{Concussion Pathophysiology}

A concussion is a transient neurological dysfunction that occurs following a biomechanical force with or without loss of consciousness, resulting in physical, cognitive, and emotional deficits including; headache, dizziness, neck pain, sleep-wake disturbances, visual difficulties, difficulty with memory, attention, concentration, problem solving and executive functioning, depression, anxiety, irritability, mood changes and post-traumatic stress (Bergman \& Bay, 2010). Due to the inability to detect anatomical abnormalities on imaging and the ability of the deficits to resolve over time, some suggest that a concussion is a temporary neuronal dysfunction rather than cell death (Mapstone, 2016).

A concussion causes changes in the neurons referred to as a neurometabolic cascade (Mapstone, 2016). Immediately after injury to the brain there is a rapid release of neurotransmitters including glutamine and N-methyl-D-aspartate which causes 
unchecked ionic fluxes of calcium and sodium entering the cell and potassium exiting the cell. In an attempt to restore ionic balance, the sodium potassium adenosine triphosphate pumps go into overdrive, triggering a dramatic jump in glucose metabolism. Giza \& Hovda (2001) note that there is a disparity between glucose supply and demand and in the setting of diminished blood flow this leads to a cellular energy crisis.

According to the CDC (2015), concussion symptoms fall into four categories: thinking/remembering, physical, emotional/mood, and sleep disturbances. Thinking or cognitive deficits include difficulty thinking clearly, concentrating, remembering new information or feeling slowed down. Physical deficits include headache, blurred vision, nausea or vomiting, dizziness, sensitivity to light and noise, balance problems or fatigue. Concussion patients may experience mood disturbances including irritability, depression, anxiety or heightened emotions. Sleep may also become effected leading to sleeping more or less than usual and trouble falling asleep. The Brain Trauma Foundation and the American Association of Neuroscience Nurses identify similar common signs and symptoms. On average most people with concussion recover quickly and fully; however, in some people it may take weeks, months or longer to recover (CDC, 2015).

It is important to point out that research on concussion pathophysiology has been conducted on animals and then applied to humans. It is essential for healthcare providers to understand the basic pathophysiology of concussion because it highlights the importance of cognitive and physical rest after injury, as well as the risk of reinjuring the brain. It should also be noted that concussion and MTBI mean the same thing and will be used interchangeably in this proposal. The following proposal is aimed toward nurses 
working in an adult ED so current pediatric concussion guidelines will not be discussed here.

\section{Prognosis}

In 2004, the World Health Organizations (WHO) Collaborating Centre Task Force on Mild Traumatic Brain Injury performed a systematic review on the prognosis for the individual with MTBI. The main objective of this task force was to find the best evidence on the epidemiology, diagnosis, treatment and prognosis related to MTBI. They found 120 articles pertaining to the prognosis of MTBI, of which studies 66 pertained to the prognosis of MTBI in adults, the others pertained to children and are not discussed here.

There is consistent evidence suggesting cognitive deficits in adults are present in the acute phase and largely resolve within 3 months, but may last up to a year. No studies found a connection between loss of consciousness (LOC) and increased cognitive deficits following MTBI, therefore LOC is not a requirement in order to diagnose a concussion. Mortality rates after MTBI range from $0 \%$ to $0.9 \%$ and are considered rare (Carroll et al., 2004).

A prospective dual-cohort study by Kraus, Schaffer, Ayers, Stenehjem \& Shen (2005) compared patients with a physician-diagnosed MTBI with a comparison cohort of patients with other physician-diagnosed injuries comparable in severity but not involving the head (fractures, contusions, lacerations or strains). Each cohort consisted of 235 patients. The MTBI cohort most commonly reported fatigue (43\%), weakness (43\%), memory problems (40\%), headache (36\%), and dizziness (34\%) at 6 months' post injury. 
While the comparison cohort most commonly reported weakness (46\%) and fatigue (43\%) (Kraus, Schaffer, Ayers, Stenehjem, \& Shen, 2005). Patients in the MTBI cohort used rehabilitation (23\%), counseling (26\%), physician (12\%) and acute care services (14\%) (Kraus et al., 2005). Kraus et al. (2005) also found that $82.6 \%$ of the MTBI cohort reported one or more symptoms during the 6 months follow up period.

In a systematic review by Carroll et al. (2014), twenty-one articles about the prognosis after MTBI in adults in regard to cognitive, psychiatric and mortality outcomes were critically reviewed. This review found evidence of MTBI associated cognitive deficits between 48 hours and 2 weeks; however, the deficits and their magnitude varied (Carroll et al., 2014). Carroll et al. (2014), also found that cognitive deficits may persist longer than 3 months in some populations, but again, there was no consistency on the exact deficits present. Furthermore, it was found that LOC at the time of injury is unrelated to later cognitive deficits. Carroll et al. (2014) noted that large confirmatory studies should be conducted tracking concussion recovery over an extended period of time to reach firm conclusions regarding prognosis.

Three articles in the systematic review addressed suicide and psychiatric outcomes following MTBI (Carroll et al.,2014). One study found increased prevalence of psychiatric illness in the first year following MTBI and that risk persisted through the first 2 years post injury (Fann et al., 2004). Another study found an increased risk of schizophrenia among men within 5 years post-MTBI; the risk was greatest within the first year (Nielsen et al., 2002). Finally, one study found the incidence of suicide post-MTBI to be 3 times that of the general population, and in persons with an accompanying diagnosis of substance abuse, the risk was 5 times higher (Teasdale \& Engberg, 2001). 
Currently there is little evidence to suggest an increase in psychiatric illness post-MTBI, but clinical monitoring of mood and psychiatric status is recommended (Carroll et al., 2014). Three studies investigated mortality post-MTBI but were unable to determine if deaths were related to MTBI or other causes (Carroll et al., 2014).

Carney et al. (2014), performed a systematic review of prevalent indicators of concussion which included 231 studies that met criteria. They found consistent indicators of concussion include; observed and documented disorientation or confusion immediately after event, impaired balance within one day after injury, slower reaction time within two days of injury, and impaired verbal learning and memory within two days after injury. Impairment in cognitive function declined from $58 \%$ on day one to $8 \%$ on day seven, possibly indicating the majority of cognitive function resolves in one week (Carney et. al., 2014).

Ganti et al. (2015) preformed a retrospective chart review of adult patients presenting to the ED with MTBI to identify factors associated with an unplanned return to the ED within 72 hours, and investigate patient symptoms upon return visit. The cohort consisted of 2,567 patients between January 1, 2008 and July 3, 2011 at a level I trauma center in the southeastern United States. They found that 124 patients (approximately 5\%) returned to the ED within a 72-hour time period. Determinants of return ED visit include male gender (66.9\%), black race (22\%), patients with an intracranial bleed on head CT (74.5\%), motor vehicle collision (MVC) and a lower prehospital Glasgow Coma Score (GCS) (Ganti et al., 2015). Reasons for returning to the ED include post-concussion syndrome (PCS) (46\%), pain (18.7\%), 14.6\% were called 
back to the ED for evaluation when laboratory test results came back, and $9.76 \%$ returned for evaluation after a repeat head injury (Ganti et al., 2015).

Ganti et al. (2015) identified four potential areas of improvement for ED treatment of MTBI including patient education about PCS symptoms, improved pain management, retaining patients in the ED until all test results are back, and specific discharge education regarding return to normal activities. Ganti (2015) concluded that if patients are made aware of what symptoms to expect after discharge, fewer patients will return for common concussion complaints "thus conserving hospital resources and mitigating MTBI's financial burden on the patient" (pp 484). It was also identified that patients should be given specific discharge instructions that limit return to normal activities in order to reduce patients risk for recurrent head injury. This review demonstrated the need for early educational intervention and better quality discharge instructions to improve patient outcomes.

One fifth of the general population visits an emergency department each year and $87 \%$ of those patients are discharged to home. The Centers for Medicare and Medicaid Services (CMS) support compliance measures for written discharge instructions in hospitalized patients; however, ED discharge is not specifically addressed (Atzema et al., 2013). Good discharge instructions prevent repeat ED visits and subsequent hospitalizations, however the time it takes to provide thorough instructions may be difficult (Atzema et al. 2013). 


\section{Treatments}

The World Health Organization (WHO) Collaborating Centre Task Force on Mild Traumatic Brain Injury published a systematic review on the non-surgical interventions for MTBI in 2004 (Borg et al., 2004). Borg et al. (2004) found few studies that provided strong evidence on non-surgical interventions in MTBI patients. It is thought that because MTBI lacks a uniform definition, valid diagnostic testing, and poor understanding of prognostic factors, it remains difficult to interpret and implement research studies. There is also a relationship between factors unrelated to brain injury such as depression, musculoskeletal injuries, and generalized body pain which contribute to poor outcomes in individuals with MTBI, making it difficult to determine how to intervene (Borg et al., 2004). The task force conducted a new systematic review in 2012, but have not yet finished that update.

Borg et al. (2004) found evidence that providing early educational information that includes material about the injury, common complaints, coping strategies, reassurance about a good outcome, and information on how to get access to further support when needed should be given at the time of injury (Borg et al., 2004). Borg et al. (2004) also found that patients should be encouraged to become active as soon as possible following their injury because bedrest has not been shown to improve outcomes. This recommendation is based on the fact that strict bedrest has not shown to improve outcomes for this patient population and can lead to further complications. However, the CDC HEADS UP Campaign recommends getting plenty of sleep at night, rest during the day and avoiding activities that are physically demanding (physical rest) or require a lot of concentration (cognitive rest). They recommend gradually increasing activity but to 
stop and rest if symptoms worsen (CDC, 2015). In summary, the task force recommends that MTBI patients be provided with simple but comprehensive education materials and reassurance to gradually return to normal activity.

A systematic review of treatments for mild TBI was conducted and published in Brain Injury in 2005. Eighteen brain injury journals between the years 1980-2003 were manually searched as well as six electronic databases. This yielded 20 original research studies included in the systematic review by Comper, Bisschop, Carnide and Tricco (2005). Treatment interventions were categorized into four general groups: pharmacotherapy, cognitive rehabilitation, patient education, and other treatment interventions. The results are discussed in the next several paragraphs.

Pharmacologic studies explored the effects of amitriptyline, sertraline, dihydroergotamine, and desmopressin acetate on psychological symptoms associated with MTBI including headache, cognitive dysfunction, and depression (Comper et al., 2005). Eight studies evaluated pharmacological interventions in MTBI treatment with half of them evaluating the antidepressant amitriptyline for depression and headache management (Comper et al., 2005). The results of these studies were mixed failing to produce solid evidence that pharmacological treatments improve MTBI symptoms (Comper et al. 2005).

Three studies used cognitive rehabilitation interventions to attempt to improve attention, memory function and speed of cognitive processing (Comper et al., 2005). Subjects in all three studies showed improvement on neuropsychological test scores but it is unclear whether practice effects, which refers to gains on cognitive test scores due to 
persons being retested on the same instrument, had an impact on the results, producing artificially inflated scores. One study used a single group design and another failed to use a true control group, therefore lacking proper comparisons, causing uncertainty in whether improvement was seen because of the cognitive rehabilitation treatment (Comper et al., 2005). In addition, the researchers found these studies had limited generalizability of results (Comper et al., 2005).

Patient education was evaluated in seven studies where informational interventions were used including: reassurance, expected recovery times, and strategies for symptom management. Six studies were randomized control trials (RCT) and one was a control trial without randomization. According to the researchers, evidence was sufficient to recommend patient education including symptom-related information and supportive interaction and reassurance (Comper, Bisschop, Carnide, \& Tricco, 2005). Education and emotional support appeared to improve MTBI patient's somatic and psychological complaints. Furthermore, the education intervention studies were among the strongest studies methodologically in this review supporting the inclusion of these findings in practice.

Nygren-de Boussard et al. (2014) conducted a systematic review on nonsurgical interventions after MTBI in adults. Two randomized control trials were deemed scientifically admissible and were included in the review. One conducted in the U.S. (Bell et al., 2008), provided telephone counseling and education to the intervention group and the control group received standard treatment, which included verbal and written discharge instructions. Patients enrolled in the intervention group also received telephone assistance managing MTBI related symptoms. These patients were followed up at 6 
months and it was found that the intervention group had fewer symptoms of depression, anxiety, change in major role performance and participation in community activities compared with the control group, which illustrates the potential of early educational information and symptom management to improve recovery (Bell et al., 2008).

The second study was a Dutch study (de Kruijk et al., 2002), comparing the effectiveness of extended bedrest to promote a return to activities. The intervention group were assigned to 6 days of full bedrest and told to return to full activity between 7 and 11 days; the control group was encouraged to remain on bed rest for no more than 4 hours during the first day then told to return to full activity within 4-days post injury. These patients were followed up at 2 weeks and 3 and 6 months, with no statistically significant differences in reported symptoms or life satisfaction. This reinforces that extended bedrest after MTBI does not result in better recovery than return to usual activities (de Kruijk et al., 2002).

Nygren-de Boussard et al. (2014) compared their results with the WHO MTBI Task Force recommendations confirming previous findings that concussion patients should be provided with educational information early after injury and encouraged to resume regular activity. Given the low number of high quality studies, there is a need for well-designed intervention studies to develop effective treatments and improve patient outcomes.

\section{Management}

Lawler and Terregino (1996) devised practice guidelines for patients who sustained MTBI presenting in an acute care setting using a systematic approach. The 
protocol is the result of an interdisciplinary team research project involving the department of emergency medicine, trauma surgery, and neuropsychology in a large urban tertiary care center with a level I trauma designation. The evaluation protocol was designed for patients 18 years or older diagnosed with MTBI and is divided into three parts: medical assessment, cognitive assessment and assessment of high risk factors. The medical assessment included CT scanning of the head for patients with acceleration/deceleration injury or blunt trauma with loss of conciseness (LOC) or amnesia. It was recommended that patients with a Glasgow Coma Scale (GCS) score of 15 (score range 3-15) should be discharged home with a responsible adult and observed if under the influence of drugs or alcohol until the intoxicate wears off.

The educational component provided clearly written information concerning physical, cognitive, and emotional/behavioral symptoms. The goal is to avoid frustrations and unrealistic expectations for returning to school or work. Lawler \& Terregino (1996), recommend gradual return to activities and educational information about anticipated problems. Patients who require follow up after screening or develop problems after discharge are referred to the outpatient concussion clinic. The aim of the protocol is preventing neurobehavioral complications post-concussion by intervening early with education and follow up (Lawler \& Terregino, 1996). This protocol serves as a model for other healthcare providers to effectively assess and treat MTBI patients in the urgent are setting.

Jagoda et al. (2008) published a clinical policy as a part of the CDC HEADS UP campaign, which provides evidence-based recommendations regarding the management of adult MTBI patients in the acute setting. A critical appraisal of the literature was 
conducted and the evidence was presented as Level A, Level B and Level C

recommendations. A Level B recommendation according to Jagoda et al. (2008) is for "Patients with an isolated MTBI who have a negative head CT scan are at minimal risk for developing an intracranial lesion and therefore may be safely discharged from the ED” (Jagoda et al., 2008, p. 723). There remain certain subpopulations who may be at increased risk of delayed complications, such as individuals with bleeding disorders, on anticoagulant therapy, with previous neurosurgical procedures, or with significant previous neurological disease, therefore limiting this to Level B evidence.

Jagoda et al. (2008), made a Level C recommendation that MTBI patients must be discharged from the ED with information about PCS. Discharge instructions vary and most often lack information concerning possible PCS symptoms. Jagoda et al. (2008) found that PCS education can reduce long-term symptoms. Nonetheless, PCS education is considered Level C recommendation due to conflicting reports on the effectiveness of its use.

Haydel (2012) critically appraised the literature to provide an evidence-based approach to concussion in the ED. The author asserted that after careful evaluation in the ED, "patients whose neurological examination, mental status, and CT are all normal may be discharged to home" (Haydel, 2012, p. 17). However, Haydel also found MTBI patients discharged from the ED remember only $30 \%$ to $50 \%$ of verbal instructions. Frequently, cognitive function is compromised after MTBI and it is suggested that clear, written instructions be provided to family members. It is estimated that almost a third of patients will experience concussion related symptoms for up to a month after injury, which can lead to anxiety (Haydel, 2012). At discharge written as well as verbal 
instructions that include symptoms to expect after concussion, time course, overall positive prognosis, activity limitations and when to seek a neurologist or concussion specialist for further testing should be provided (Haydel, 2012). Well-written discharge instructions, such as those recommended by Haydel are provided by the CDC's HEADS UP Campaign and are readily available online for patients who have suffered MTBI.

\section{Discharge Practices in the Emergency Department}

Bay and Strong (2011) analyzed the discharge practices of ED nurses for adult MTBI patients. Specifically, they wanted to determine ED nurses' awareness of the CDC's Acute Concussion Evaluation (ACE) care plan. A descriptive cross-sectional survey design was used on a randomized sample of 500 nurses, residing in the following states; Indiana, Michigan, Wisconsin, Illinois, and Ohio. They used a nonprobability convenience sample of nurses practicing in ED setting for more than 16 hours a week. The researchers developed a survey tailored to the ACE tool. Data collection occurred in the summer of 2008 and of the 500 surveys, 87 respondents met eligibility criteria and completed the requested data.

The results indicated that a majority of the sample (71.3\%) were unaware of the CDC's ACE care plan and only $17.2 \%$ claimed that this care plan was a standard of care in their ED. This sample consists of experienced and certified ED nurses and the majority were unaware of the CDC guidelines (Bay \& Strong, 2011). Nurses claimed to provide injury-specific instructions that included return to work, pain relief, and wound care at a mean percentage of $40.18 \%$. The nurses in this survey also claimed to provide preventative discharge teaching about activity avoidance, interventions for fatigue or 
sleep problems, and stress management at a mean percentage of $37.71 \%$. About $86 \%$ of participants claimed to have a work place barrier to discharge teaching. Four main barriers were identified: lack of time (37.9\%), lack of a standardized form (17.2\%), patient lack of interest (17.2\%) and a limited resource (12.6\%). Other barriers included noisy environment, difficulty with patient flow, and lack of continuity of nurses (Bay \& Strong, 2011).

Limitations of this study include a response rate of $25 \%$ to the survey and reflect responses from experienced ED nurses who were familiar with MBTI patient care, thus awareness of the CDC's ACE guidelines may be lower than reported in this study. According to Bay \& Strong, delivering consistent discharge teaching to the MTBI patient about expected symptoms, recovery, and prevention of future injury should be more systematic and it is difficult to provide consistent information without standardized educational material. In addition, investigation of the best teaching methods in the ED is needed to overcome barriers such as time limitations and hectic environments (Bay \& Strong, 2011).

A study by Atzema et al. (2013) evaluated the effectiveness of online video discharge instructions on patient comprehension and recall of instructions. This study is a single-center, randomized control trial in the ED of a tertiary adult hospital in Toronto, Canada. The study was conducted between November 2011 and January 2012, with 133 participants contacted by telephone and asked three standardized questions about their discharge instructions and their satisfaction with the video instructions. The intervention group watched an online video about their discharge diagnosis at home in addition to 
routine ED discharge instructions and the control group received routine ED discharge instructions only.

Study limitations include a single site study with slightly lower proportion of low socioeconomic status participants and everyone had internet access. Also the research assistant was not blinded to the group. Results indicated that those in the intervention group answered all the standardized questions correctly 3.5 times higher than the control group. The intervention group also scored the videos at a rating of 10 , on a scale on 1-10, for video quality and increased understanding. The intervention group not only had improved understanding of their discharge instructions but also had a high level of satisfaction with regard to their comprehension. Given that an average ED discharge lasts 76 seconds, utilizing technology to offset communication deficiencies can achieve improved patient comprehension (Atzema et al., 2013).

Although discharge teaching provided by nurses is a "professional expectation and a Joint Commission requirement, there is no standardization for health teaching or assessing patient comprehension" (Alberti \& Nannini, 2013, p. 186). Alberti \& Nannini (2013) conducted a literature review examining patient comprehension of discharge instructions from the ED or urgent care settings. An observational study found that only $16 \%$ of providers asked if the patient had questions during discharge and none of them asked if patients understood (Alberti \& Nannini, 2013).

Upchurch et al. (2015) conducted a retrospective chart review that evaluated sports-related concussion discharge instructions and physician referrals. The objective of this study was to evaluate the effect of the CDC HEADS UP Campaign on 
appropriateness of discharge instructions. The CDC updated their recommendations in 2007 and "strongly emphasized the need for both physical and cognitive rest" (Upchurch, Morgan, Umfress, Yang, \& Riederer, 2015, p. 298). Researchers found that appropriate discharge instructions improved from $66 \%$ to $75 \%$ in 2010 , which was not statistically significant. Regardless of the new focus on cognitive rest, it was not recommended for any patients until 2008 and only 12\% in 2012 (Upchurch et al., 2015).

As indicated by this study there is a need for improvement in discharging MTBI patients from the ED. There needs to be a standardization of appropriate discharge instructions as well as education of ED nurses to promote better discharge teaching (Upchurch et al., 2015). Despite the CDC HEADS UP campaign, efforts to provide appropriate discharge instructions have not significantly improved.

A systematic review by Eliyahu, Kirkland, Campbell \& Rowe (2016) assessed the effectiveness of early educational interventions in the ED. Five studies were included in this systematic review utilizing the PRISMA methodology guidelines. It was found that two of the five studies demonstrated significant improvement in patients' PCS symptoms after receiving early educational interventions. Despite these findings, the researchers determined that there was insufficient high-quality evidence to suggest that early interventions decrease PCS symptoms and the authors made no recommendations (Eliyahu, Kirkland, Campbell, \& Rowe, 2016). Research is needed to determine what interventions are best for MTBI patients in order to produce evidence-based guidelines and improve outcomes. 
It is clear from the review of these articles that patient education is an important aspect of care for the concussion population; however, more research is needed to determine how best to deliver education to the MTBI population; as well as improving nursing awareness of evidence-based guidelines. Symptom management is the gold standard for concussion treatment and needs to be included in discharge instructions, not only to alleviate patient anxiety, but to prevent unnecessary return visits to the ED. The CDC HEADS UP Campaign strongly recommends both physical and cognitive rest with gradual return to activity, which is congruent with current research. A major barrier to patient discharge education in the ED is a lack of time with a wide variation in instructions which require uniformity and alignment with current guidelines. Most importantly, ED nurses need further concussion education to be knowledgeable patient educators. 


\section{Theoretical Framework}

Malcolm Knowles’ Adult Learning Theory was the theoretical framework selected to guide this program development project. Knowles' theory is a learning theory that was developed for the specific needs of adults and is based on six principles: the need to know, self-concept, experience, readiness to learn, orientation to learning and motivation (Knowles, Holton, \& Swanson, 2011). Adults need to know why knowledge is important and how it can be applied to their life. Self-concept refers to an adult becoming more self-directed and independent as he or she matures. Adults typically want to choose what they learn, when they learn it, and how they learn it. Adult learners have a wealth of life experiences that they bring with them into new learning experiences and learn better when these experiences are acknowledged. Whether or not an adult is ready to learn depends on what they need to know to solve real-life problems. Adults are problem-oriented learners and are compelled to learn when presented with specific life situations. External motivators will compel adults to learn but the more potent motivators are internal. Adults are typically motivated by the need to solve practical and immediate life problems (Knowles et al., 2011).

Nursing, like many other professions, requires lifelong learning in order to maintain competencies and knowledge within their field. Nurses are adult learners and are motivated to learn when it directly relates to their clinical practice (Knowles et al., 2011). Rather than assuming the usual role of lecturer, educators can take on the function of a resource person or facilitator, giving control to the adult learner. With a thorough understanding of Knowles' theory, educators can ensure engagement of the students and provide a quality learning experience. 
Nursing is a complex, ever changing profession and there is a need to keep up-todate on the latest healthcare information and practices. Nurses have a variety of resources available to them including text books, professional journals, drug references, websites, classes, certifications and evidence-based hospital protocols to keep their practice up-to-date. Developing a tailored educational program for nurses is an effective and appropriate way to help nurses obtain new knowledge. Applying Knowles' Adult Learning Theory to an educational program allows the nurse to learn at their own convenience in a busy acute care setting.

Nurses working in the ED have a need to stay up-to-date on evidence based treatments including current concussion recommendations. Evidence supports the effectiveness of early patient education as the most important and cost effective strategy for concussion patients (Comper, Bisschop, Carnide, \& Tricco, 2005). With $80 \%$ of those with TBI treated and released directly from the ED, nurses are in a position to improve patient outcomes by providing evidence-based discharge instructions to concussion patients (Bay \& Strong, 2011). 


\section{Method}

\section{Purpose}

The purpose of this project was to evaluate the impact of an educational program on emergency department (ED) nurses' knowledge of concussion/MTBI and the current CDC recommended education for patients and families discharged home.

\section{Design}

The design of this project is a program development for quality improvement utilizing a pre-test, intervention and post-test framework to evaluate ED nurses’ knowledge of concussion and current recommend discharge education.

\section{Sample/Site}

This project uses a nonprobability convenience sampling method. The participants are nurses who work in the ED at Rhode Island Hospital (RIH). RIH is a 719 bed level one trauma center in Providence, Rhode Island. The potential sample size was approximately 180 nurses. The goal was to recruit at least $30 \%$ or 45 nurses to participate in the study. Inclusion criteria consisted of all nurses working in the RIH ED who were willing to participate. Any ED nurse regardless of years of experience or education level would benefit from this educational program. Non-nursing personnel were excluded from this sample.

\section{Procedures}

This project was conducted by the author, who was also the program developer, with support from the primary investigator, Cynthia Padula. Prior to beginning this 
project, the program developer obtained approval from Rhode Island College and Lifespan internal review boards' (IRB). Permission was obtained from the Clinical Nurse Manager and Clinical Nurse Specialist (CNS) of the RIH ED. An informational letter via e-mail explaining procedures was sent out one week prior to program implementation (Appendix A). Nurses were asked to anonymously complete an online, author developed pre-test using an online survey platform called SurveyMonkey via work e-mail (Appendix $B)$. The nurses were asked to use their mother's birthdate as an identifier on the pretest. The pre-test was available for nurses to complete over a two-week period and e-mail reminders were sent out on days six and ten. Online pre-tests were closed after the twoweek period and collected by the researcher. A copy of the ten question survey can be found in Appendix $C$.

Nurses were asked to anonymously complete an online post-test via work e-mail after reviewing the author developed, MTBI education poster board that was placed in the break room and displayed for two weeks. There was also an educational packet e-mailed to the nurses containing the same information as the poster board. A copy of the poster board/informational packet can be found in Appendix $D$. The participants again were asked to use their mother's birthdate as an identifier on the post test. The poster board and post-test were available for two weeks with e-mail reminders on day six and ten. As an incentive, participants were entered into a raffle for a \$25 gift card; mention of this incentive was included in all emails sent to the ED nurses. 


\section{Data Analysis}

Descriptive statistics including total scores, mean scores and percentiles were calculated comparing pre- and post-test scores on survey monkey, completion rates and missing data.

\section{Development of the Educational Intervention}

The concussion educational program was developed and implemented using W.F. Kellogg Foundation Logic Model (W.K. Kellogg Foundation, 2004). The Logic Model is a framework that identifies outcomes and anticipates ways to measure them by providing a clear map of the project prior to development. When the project is mapped out ahead of time it is less likely to unintentionally stray off course. The components of the Logic Model consist of: resources/inputs, activities, outputs, outcomes, and impact. The Logic Model was used to develop and evaluate the effectiveness of the concussion educational program.

Resources or inputs include the human, financial, organizational and community resources available for carrying out programs activities. The most significant resource needed for this program development is the nurses' time to participate in the program. The program developer obtained approval and support from the ED Clinical Nurse Manager and CNS.

A focused needs assessment was conducted. Several nurses working in the emergency department were interviewed and it was determined that there was an interest in a concussion educational program. Rhode Island Hospital is a 719-bed acute care hospital; the largest hospital and the only level I trauma center in Rhode Island. The ED 
is one of the busiest in the Northeast with a volume of 105,000 patients each year.

Common presenting injuries include falls and motor vehicle collisions (MVC), which are two of the most common mechanisms of injury for concussion.

Furthermore, the literature supports early educational interventions at the time of injury improve patient outcomes and indicates a lack of awareness of current concussion guidelines among ED nurses. This supports the need for a concussion educational program to educate ED nurses who have the ability to improve patient outcomes through their discharge teaching.

Activities are the processes, tools, events, and actions that are used to bring about a program's intended changes or results. The program developer created educational materials which included a poster board and educational packet from the literature review, clinical experience, and the CDC HEADS UP Campaign. Malcolm Knowles' Adult Learning Theory guided this program development in the following ways. The nurses learned at their own pace and at a time that was conducive to learning. The poster board was displayed in the break room and the educational packet e-mailed to each nurse which allowed them to read it at a time convenient to them. Time is a valuable commodity in a fast-paced, busy ED and it is important to make information easily and readily accessible to staff nurses.

Outputs are the direct products of a program's activities and may include types, levels, and targets of services to be delivered by a program. The expected output of this program development was increased knowledge of concussion. Knowledge was measured with a ten question, computer-based multiple choice exam. There is currently 
no measurement tool found in the literature to assess nursing knowledge of concussion.

Therefore, this test was developed by the program developer and approved by the RIH ED CNS. Following this, the test was piloted with a comparable sample of registered nurses to test for clarity. The program content and objectives are displayed in Table 1.

Table 1.

Concussion Program Development

\begin{tabular}{|l|l|}
\hline \multicolumn{1}{|c|}{ Content } & \multicolumn{1}{c|}{ Objectives } \\
\hline $\begin{array}{l}\text { What is a concussion? } \\
\text { Common signs and symptoms }\end{array}$ & $\begin{array}{l}\text { The nurse will be able to define } \\
\text { concussion and name the common signs } \\
\text { and symptoms. }\end{array}$ \\
\hline Concussion pathophysiology & $\begin{array}{l}\text { The nurse will understand basic } \\
\text { pathophysiology to highlight the } \\
\text { importance of cognitive and physical rest. }\end{array}$ \\
\hline Concussion prognosis & $\begin{array}{l}\text { The nurse will understand the likely } \\
\text { course of concussion and post-concussive } \\
\text { syndrome. }\end{array}$ \\
\hline Concussion treatment & $\begin{array}{l}\text { The nurse will be knowledgeable about } \\
\text { current treatment recommendations } \\
\text { including patient education, return to } \\
\text { activity, cognitive \& physical rest and } \\
\text { reassurance. }\end{array}$ \\
\hline $\begin{array}{l}\text { Discharge education } \\
\text { What to expect } \\
\text { Warning signs } \\
\text { Returning to daily activity and work } \\
\text { Follow up }\end{array}$ & $\begin{array}{l}\text { The nurse will be able to identify the key } \\
\text { elements necessary to inform patients } \\
\text { diagnosed with concussion and families } \\
\text { prior to discharge from the ED setting. }\end{array}$ \\
\hline
\end{tabular}

Outcomes are expected changes in the population served that result from a program's activities and fall along a continuum, ranging from short to long term results. The primary outcome of this program was to increase ED nurses' knowledge of concussion/MTBI and the current CDC recommended education for patients and families 
discharged home. A long-term outcome would be to improve nursing discharge instructions to concussion patients and families in the ED.

Impacts are the changes expected at an organizational, community or systems level. Nurses with increased knowledge regarding concussions have the potential to improve patient's outcomes with their discharge teaching, leading to the improved health status of local communities affected by concussion. Improved discharge teaching also has the potential to prevent unnecessary return visits to the ED reducing healthcare costs.

It is paramount that scholarly projects uphold ethical principles including beneficence, non-maleficence, respect for autonomy, fairness, truthfulness and justice. Human rights are basic rights and freedoms inherent to all human beings regardless of nationality, race, religion, sex or any other status. Ethics and human rights are complementary and the combined use maximizes protection of patient rights. This program development for ED nurses falls in line with these basic ethical and human rights beliefs. Participation in this project was voluntary and caused no harm. Neither the pre- nor the post-test included identifiable information and the results were accessed using a password protected computer. 


\section{Results}

The target population for this program development was nurses working in the ED at RIH $(n=180)$ at the time of the intervention. Out of the potential sample size 51 nurses $(28 \%)$ completed the pre-test and scores ranged from $30 \%$ to $90 \%$, with a mean score of $61.96 \%$. Thirty-seven percent of the nurses scored $50 \%$ or less on the pretest, $55 \%$ scored $60-80 \%$, and $8 \%$ scored $>90 \%$. Appendix E illustrates individual pre-test results by question and overall score.

Twenty-eight nurses (15\%) completed the post-test with a range of $40 \%$ to $100 \%$ and a mean score of $78.2 \%$. Seven percent of the nurses scored $50 \%$ or less on the post test, $57 \%$ scored $60-80 \%$, and $36 \%$ scored $>90 \%$. Appendix $F$ illustrates individual posttest results by question and overall score.

The most improved question from pre-test to post-test was related to mechanism of injury (Q3), 19.61\% of nurses answered correctly on pre-test and 89.29\% answered correctly on post-test. Question 1 pertains to the definition of concussion and was answered correctly $90.20 \%$ on the pretest compared to $100 \%$ correct on the post-test. Two questions were related to diagnosis of concussion (Q2, Q4), with $66.67 \%$ to $82.35 \%$ correct on the pre-test and $92.59 \%$ to $96.43 \%$ correct on the post-test respectively. Question 6 was related to treatment and the scores improved from $60.78 \%$ to $89.29 \%$; identifying symptoms (Q9) improved from $13.73 \%$ to $42.86 \%$.

Important discharge education pertained to questions 8 and 10. Question 10 scores improved from $64.71 \%$ to $92.86 \%$. Question 8 actually decreased from $52.94 \%$ to $50 \%$, 
as did scores for defining post-concussive syndrome (Q7) which decreased from $80.39 \%$ to $66.67 \%$. Summary and conclusions will be discussed next. 


\section{Summary and Conclusions}

A concussion or MTBI is the most common type of TBI and accounts for $75 \%$ of individuals presenting to ED with head trauma (Bergman \& Bay, 2010). Despite the designation, MTBI can lead to significant physical, cognitive and emotional impairment. According to the CDC (2015), concussion symptoms fall into four categories: thinking/remembering, physical, emotional/mood and sleep. On average most people with concussion recover quickly and fully but in some people it may take weeks, months or longer to recover (CDC, 2015). Up to $30 \%$ of patients discharged with a diagnosis of concussion have symptoms at three months and $15 \%$ are symptomatic one-year post head injury (Haydel, 2012). These persistent symptoms are referred to as post-concussion syndrome and often lead to missed work and decreased productivity as well as increasing health care costs. According to Haydel (2012), TBI exceeds $\$ 60$ billion annually in the United States including direct as well as indirect costs. The impact of early interventions on PCS can lead to decreased morbidity and healthcare cost.

There is no specific treatment for concussion, therefore focus is on managing symptoms. It is clear from the literature review that patient education is an important aspect of care for the concussion population. Symptom management is the gold standard for concussion treatment and must be included in discharge instructions, not only to alleviate patient anxiety but to prevent unnecessary return visits to the ED. The CDC HEADS UP Campaign strongly recommends both physical and cognitive rest with gradual return to activity and is congruent with current research.

One fifth of the population visits an emergency department each year and $87 \%$ of those patients are discharged to home. Good discharge instructions prevent repeat ED 
visits and subsequent hospitalizations (Atzema et al. 2013). Nurses working in the ED have a need to stay up-to-date on evidence based treatments including current concussion management recommendations. Evidence supports the effectiveness of early patient education as the most important and cost effective strategy for concussion patients (Comper, Bisschop, Carnide, \& Tricco, 2005). With $80 \%$ of those with TBI treated and released directly from the $\mathrm{ED}$, nurses are in a position to improve patient outcomes by providing evidence-based discharge instructions to concussion patients at ED discharge (Bay \& Strong, 2011).

The purpose of this project was to evaluate the impact of an educational program on emergency department (ED) nurses' knowledge of concussion/MTBI and the current CDC recommended education for patients and families discharged home. The design of this project was a program development for quality improvement with pre-test, intervention and post-test to evaluate ED nurses' knowledge of concussion. There is currently no measurement tool found in the literature to assess nursing knowledge of concussion. Therefore, a ten question test was developed by the author and piloted on a comparable sample. Nurses were asked to anonymously complete an online, author developed pre-test and post-test using an online survey platform called SurveyMonkey sent to them via work e-mail (Appendix B). A poster board was displayed in the break room and informational packet e-mailed to every nurse; they were asked to review educational material at their own convenience.

The target population for this program development was approximately 180 nurses who work in the emergency department at Rhode Island Hospital. Out of the potential sample size 51 nurses (28\%) completed the pre-test with a mean score of 
$61.96 \%$. Twenty-eight nurses $(15 \%)$ completed the post-test with a mean score of $78.2 \%$, which is an overall improvement of $16.24 \%$. The most improved score was on mechanism of injury. The majority of nurses thought that sports-related injury was the most common cause of concussion prior to participating in the educational program. After completing the program, $100 \%$ of the nurses could correctly define concussion; scores also improved on questions pertaining to diagnosis and treatment. Some scores, however, decreased on post-test, specifically those which pertained to defining PCS (Q7) and one of the two questions related to discharge education (Q8). This may have been due to the question format which was used; a multiple-multiple choice question requiring the participant to select all the correct responses in order to get points for those particular questions. Most of the participants who answered these multiple-multiple questions wrong, selected most of the correct choices but failed by omitting one correct choice.

Several limitations were acknowledged including; low participation, the goal was for $30 \%$ participation of the 180 nurses $(n=54)$. Although nurses showed interest and enthusiasm for the project, there was a lack of participation. Lack of time to complete the intervention and the surveys may have been a contributor to the low participation. The method to announce the research project was also identified as a limitation. The procedure stated that all ED nurses were e-mailed at the beginning of the project then again on day six and ten for both the pre-test and post-tests. In a large health care institution, nurses get a considerable influx of daily e-mails and may have deleted or skipped over the e-mails. Some nurses may not check their work e-mail often or at all. The program developer could have incorporated different ways to raise awareness of the project such as announcements during staff meetings or flyers posted in the department. 
It is also possible that nurses reviewed the poster board and informational packet but did not participate in the survey. Anecdotally, there was increased discussion of concussion education on the unit. Another limitation was that this was a single site study involving a convenience sample. Furthermore, not all of the participants that took the pre-test went on to take the post-test and vice versa.

In conclusion, the primary outcome of this educational intervention to improve nurses' knowledge was met. The intervention was carefully crafted using the literature and clinical experience; the use of a poster board and handout allowed nurses working in a busy ED the flexibility to review the material when they had the time. A long-term outcome would be to improve nursing discharge instructions to concussion patients and families in the ED. With institutional support, this program could potentially result in improved nursing knowledge as well as improved outcomes for patients. Next recommendations and implications for advanced nursing practice are discussed. 


\section{Recommendations and Implications for Advanced Nursing Practice}

Advanced Practice Registered Nurses (APRNs) in all health care settings have the opportunity to identify knowledge deficits in clinical practice. An educational needs assessment can effectively drive change in teaching strategies to increase nurses' knowledge in the acute care setting. The APRN is in a position to stimulate change by developing an educational program with nursing staff which may enable nurses to provide more competent and evidence-based patient care to improve patient outcomes.

It was clear from the literature review that research is needed to determine what interventions are best for MTBI patients in order to produce evidence-based guidelines and improve outcomes. The APRN can be involved in areas of concussion research in various populations expanding current knowledge of concussion and developing improved evidence-based guidelines. In addition, the APRN is capable of leading an interdisciplinary team to develop evidence-based guidelines for not only the treatment of concussion, but for patient discharge instructions. Clearly defined guidelines have the potential to decrease variation in practice and discharge teaching. APRN presence on a unit can ensure that these guidelines are utilized. Given that the CDC has already developed evidence-based discharge instructions for concussion, the APRN can use these as a starting point when developing standardized discharge instructions for their healthcare institution. Fall prevention and motor vehicle safety programs should also be implemented in the ED, considering that they are the leading cause of concussions. Prevention of these accidents in the future will improve the overall health of the community, decreasing the incidence of MTBI. 
Despite the CDC HEADS UP campaign efforts, appropriate discharge instructions have not significantly improved. More work is needed to improve healthcare providers' knowledge base of concussions and the evidence-based discharge instructions already available by the CDC. Given the anecdotally noted increase in discussion about concussion education on this particular unit, it would be interesting to see if future concussion educational programs would generate improved participation. This would be an ideal opportunity for an APRN to institute an educational program for staff. The APRN is in a position to have a remarkable impact on the future of our healthcare system and will be sought out for ability to improve healthcare outcomes. 


\section{References}

Alberti, T. L., \& Nannini, A. (2013). Patient comprehension of discharge instructions from the emergency department: a literature review. Journal of the American Association of Nurse Practitioners, 25, 186-194. http://dx.doi.org/10.1111/j.17457599.2012.00767.x

Atzema, C. L., Austin, P. C., Wu, L., Brzozowski, M., Feldman, M., McDonnell, M., \& Mazurik, L. (2013, November). Speak fast, use jargon and don't repeat yourself: A randomized trial assessing the effectiveness of online videos to supplement emergency department discharge instructions. PLOS ONE, 8(11). http://dx.doi.org/10.1371/journal.pone.0077057

Bay, E., \& Strong, C. (2011). Mild traumatic brain injury: A midwest survey of discharge teaching practices of emergency department nurses. Advanced Emergency Nursing Journal, 33, 181-192. http://dx.doi.org/10.1097/TME.0b013e318217c958

Bell, K. R., Hoffman, J. M., Temkin, N. R., et al. (2008). The effect of telephone counseling on reducing post-traumatic symptoms after mild traumatic brain injury: a randomized trial. Journal Neurol Neurosurg Psychiatry. 79, 1275-1281.

Bergman, K., \& Bay, E. (2010, May). Mild traumatic brain injury/concussion: a review of ED nurses. Journal of Emergency Nursing, 36(3), 221-230. http://dx.doi.org/10.1016/j.jen.2009.07.001

Borg, J., Holm, L., Peloso, P. M., Cassidy, D., Carroll, L. J., Holst, H. V., ... Yates, D. (2004). Non-surgical intervention and cost for mild traumatic brain injury: results of the WHO collaborating centre task force on mild traumatic brain injury. 
Journal of Rehabilitation Medicine, 43, 76-83.

http://dx.doi.org/10.1080/16501960410023840

Carney, N., Ghajar, J., Jagoda, A., Bedrick, S., Davis-O’Reilly, C., du Coudray, H.,...Riggio, S. (2014). Concussion guidelines step 1: systematic review of prevalent indicators. Neurosurgery, 75(3), 3-15.

http://dx.doi.org/10.12227/NEU.0000000000000433

Carroll, L. J., Cassidy, D., Cancelliere, C., Cote, P., Hincapie, C. A., Kristman, V. L., ... Hartvigsen, J. (2014). Systematic review of the prognosis after mild traumatic brain injury in adults: cognitive, psychiatric, and mortality outcomes: results of the international collaboration on mild traumatic brain injury prognosis. Archives of Physical Medicine and Rehabilitation, 95, 152-173. http://dx.doi.org/http://dx.doi.org/10.1016/j.apmr.2013.08.300

Carroll, L. J., Cassidy, D., Peloso, P. M., Borge, J., Holst, H. V., Holm, L., ... Pepin, M. (2004). Prognosis for mild traumatic brain injury: results of the WHO collaborating centre task force on mild traumatic brain injury. Journal of Rehabilitation Medicine, 43, 84-105.

http://dx.doi.org/10.1080/16501960410023859

Centers for Disease Control and Prevention. (2015). http://www.cdc.gov/headsup/basics/concussion_whatis.html

Comper, P., Bisschop, S. M., Carnide, N., \& Tricco, A. (2005, October). A systematic review of treatments for mild traumatic brain injury. Brain Injury, 19(11), 863880. http://dx.doi.org/10.1080/02699050400025042 
de Kruijk, J. R., Leffers, P., Meerhoff, S., Rutten, J., Twijnstra, A., (2002). Effectiveness of bed rest after mild traumatic brain injury: a randomized control trial. Journal of Neurol Neurosurg Psychiatry, 73, 167-172.

Eliyahu, L., Kirkland, S., Campbell, S., \& Rowe, B. H. (2016, May). The effectiveness of early educational interventions in the emergency department to reduce incidence or severity of post-concussion syndrome following a concussion: a systematic review. Academic Emergency Medicine, 23(5), 531-542. http://dx.doi.org/10.1111/acem.12924

Fann, J. R., Burington, B., Leonetti, A., Jaffe, K., Katon, W.J., Thompson, R. S., (2004). Psychiatric illness following traumatic brain injury in an adult health maintenance organization population. Arch Gen Psychiatry, 61, 52-61.

Ganti, L., Conroy, L., Bodhit, A., Shashikant Patel, P., Ayala, S., Kuchibhotla, S., ... Lottenberg, L. L. (2015, May). Understanding why patients return to the emergency department after mild traumatic brain injury within 72 hours. Western Journal of Emergency Medicine, XVI (3), 481-485. http://dx.doi.org/10.5811/westjem.2015.2.23546

Giza, C. C., \& Hovda, D. A. (2001, September). The neurometabolic cascade of concussion. Journal of Athletic Training, 36(3), 228-232. Retrieved from www.journalofathletictraining.org

Haydel, M. (2012, September). Management of mild traumatic brain injury in the emergency department. Emergency Medicine Practice, 14(9), 2-24. Retrieved from www.ebmedicine.net 
Jagoda, A. S., Bazarian, J. J., Bruns, J. J., Cantrill, S. V., Gean, A. D., Kunz Howard, P., ... Whitson, R. R. (2008, December). Clinical Policy: Neuroimaging and decision making in adult mild traumatic brain injury in the acute setting. Annals of Emergency Medicine, 52(6), 714-748. http://dx.doi.org/10.1016/j.annemergmed.2008.08.021

Knowles, M. S., Holton III, E. F., \& Swanson, R. A. (2011). The adult learner: the definitive classic in adult education and human resource development (7th ed.). Burlington, MA: Elsevier.

Kraus, J., Schaffer, K., Ayers, K., Stenehjem, J., \& Shen, H. (2005, May/June). Physical complaints, medical services use and social and employment changes following mild traumatic brain injury. Journal of Head and Trauma Rehabilitation, 20(3), 239-256. Retrieved from http://journals.lww.com/headtraumarehab/Pages/default.aspx

Lawler, K. A., \& Terregino, C. A. (1996, December). Guidelines for evaluation and education of adult patients with mild traumatic brain injuries in an acute care hospital setting. Journal of Head Trauma Rehabilitation, 11(6), 18-28.

Mapstone, P. (2016, April). Sport-related concussion in youth: a curriculum for advanced practice nurses. The Journal for Nurse Practitioners, 12(4), 250-257. http://dx.doi.org/http://dx.doi.org/10.1016/j.nurpra.2015.11.004

Nielsen, A. S., Mortensen, P. B., O’Callaghan, E., Mors, O., Ewald, H. (2002). Is head injury a risk factor for schizophrenia? Schizophr Res, 55, 93-98.

Nygren-de Boussard, C., Holm, L. W., Cancelliere, C., Godbolt, A., Boyle, E., Stalnacke, B., ... Borge, J. (2014). Nonsurgical interventions after mild traumatic brain 
injury: a systematic review. Results of the international collaboration on mild traumatic brain injury prognosis. Archives of Physical Medicine and Rehabilitation, 95, 257-264.

http://dx.doi.org/http://dx.doi.org/10.1016/j.apmr.2013.10.009

Sheedy, J., Geffen, G., Donnelly, J., \& Faux, S. (2006). Emergency department assessment of mild traumatic brain injury and prediction of post-concussion symptoms at one month post injury. Journal of Clinical and Experimental Neuropsychology, 28, 755-772. http://dx.doi.org/10.1080/13803390591000864

Teasdale, T. W., Engberg, A. W. (2001). Suicide after traumatic brain injury: a population study. Journal of Neurol Neurosurg Psychiatry, 71, 436-440.

Upchurch, C., Morgan, C. D., Umfress, A., Yang, G., \& Riederer, M. F. (2015, May). Discharge instructions for youth sports-related concussions in the emergency department, 2004 to 2012. Clinical Journal of Sports Medicine, 25(3), 297-299. Retrieved from www.cjsportmed.com W.K. Kellogg Foundation. (2004). Logic Model development Guide. Retrieved from www.wkkf.org 


\section{Appendix A}

\begin{tabular}{|ll|}
\hline IRB Approval: & $11 / 30 / 2016$ \\
\hline IRB Accepted: & $11 / 30 / 2016$ \\
\hline
\end{tabular}

Informational Letter

You are being asked to take part in a quality improvement project called Concussion: Improving Nurses' Knowledge in the Emergency Department, the purpose of this project is to increase nurses' knowledge of concussion/MTBI and the current $\mathrm{CDC}$ recommended education for patients and families discharged home. Danielle Marsden, a student at Rhode Island College and an employee of Lifespan is conducting this project in conjunction with the Principal Investigator, Cynthia Padula.

If you agree to participate, you will be asked to complete a ten question online pre-test. You will then be asked to review a poster board displayed in the breakroom that includes information about concussion and/or an informational packet that will be sent to you via lifespan email. After reviewing these items, you will be again asked to complete an online post-test. The total time to complete all of these activities is about 25 minutes.

There are no questions that should cause you any discomfort. Your taking part in this project is completely voluntary. If you do not want to participate, you are free to choose not to and may withdraw your participation at any time.

Your participation in this project may increase your knowledge regarding concussions. Neither the pre nor post test will include identifiable information; however, you will be asked to use your mother's date of birth as an identifier. The pre and post tests will be administered using an online survey platform called SurveyMonkey. The program developer, Danielle Marsden will be the only one to have access to the pre and post test results. The results will be accessed using a 
password protected computer and then logging into SurveyMonkey using another confidential password.

If you have any questions about this quality improvement project, please feel free to contact Danielle Marsden at 774-281-3285, or at dmarsden1@lifespan.org. You may also contact the Principle Investigator, Cynthia Padula, PhD, RN at 456-9720 or cpadula@ric.edu. You may also wish to contact Margaret Mock at 401-456-2775 or mmock@ric.edu. You may also contact the IRB Chair designate at IRB@ric.edu.

If you have any questions about your rights as a research subject please feel free to call our Office of Research Administration manager, Janice Muratori at 444-6897.

Thank you very much for your time!

Danielle Marsden RN, BSN

Rhode Island Hospital 


\section{Appendix B}

\begin{tabular}{|ll|}
\hline IRB Approval: & $11 / 30 / 2016$ \\
\hline IRB Accepted: & $11 / 30 / 2016$ \\
\hline
\end{tabular}

E-mail Script

Hello, my name is Danielle Marsden and I am a graduate student at Rhode Island College and also a Lifespan employee. As part of my studies I am conducting a research educational program for emergency department nurses. I will be conducting this research project in conjunction with the principle investigator, Cynthia Padula. It's called Concussion: Improving Nurses' Knowledge in the Emergency Department.

First, I will ask you to take a 10 question pre-test on SurveyMonkey. This should only take you 5-10 minutes to complete. It will test your current knowledge about concussion. This pre-test will be posted for a 2-week period. Click on link here to be brought directly to online pre-test. (insert link here) You will need to enter your mothers' birthday as an identifier, answer the 10 questions and then click done.

Then a poster board will be displayed in the break room and an educational packet sent to your e-mail. I will ask that you review the content.

Last, I will ask you to take a 10 question post-test on SurveyMonkey. Again this should only take you 5-10 minutes to complete. It will test your knowledge about concussion after teaching intervention. This post-test will also be posted for a 2-week time period.

Your participation is of course voluntary. Anyone, who participates will be entered into a drawing for a \$25 Au Bon Pain gift card.

Thank you for your time!

Danielle Marsden RN, BSN

Rhode Island College 


\section{Appendix C}

\section{Concussion Survey for Emergency Department Nurses}

Pre and Post Test

1. A concussion is a mild traumatic brain injury.

True or False

2. Most concussions result in a loss of consciousness.

True or False

3. What is the leading cause of concussion?
A. Falls
B. Sports related injuries
C. Assault
D. Motor vehicle collision

4. How is a concussion identified?
A. By looking at CT or MRI scans
B. By watching for signs and symptoms
C. By mechanism of injury and LOC
D. Both $\mathrm{A} \& \mathrm{C}$

5. Why is cognitive and physical rest important after a concussion?
A. There is a disparity between glucose supply and demand in the setting of diminished cerebral blood flow
B. The risk of reinjuring the brain while it is in cellular energy crisis is greater
C. Cognitive and physical rest are no longer recommended
D. Both A \& B

6. What is the most effective treatment for concussion?
A. Patient education \& support
B. Cognitive rehabilitation
C. Amitriptyline
D. Bed rest

7. What is post-concussive syndrome?
A. Clinical state with 3 or more symptoms
B. Persistence of symptoms for 3 months or more
C. A complication of a concussion 
D. All of the above

8. Which of the following are warning signs to teach patients to return the emergency department right away? (pick all that apply)

A. Headache that is relieved with Acetaminophen

B. Difficulty concentrating at work or school

C. Increasing confusion

D. Slurred speech

E. Headaches that worsen

9. A concussion is a transient neurological dysfunction that results in which of the following? (pick all that apply)
A. Cognitive dysfunction
B. Physical ailments
C. Emotional/mood disorder
D. Neuronal cell death
E. Sleep disturbances

10. Concussion education should include all the following EXCEPT.
A. What to expect
B. Symptom management/coping
C. Follow up
D. Bed rest
E. Activity limitations 


\section{Appendix D}

\section{Concussion Educational Program and Poster Content}

- A concussion is a type of traumatic brain injury — or TBI — caused by a bump, blow, or jolt to the head or by a hit to the body that causes the head and brain to move rapidly back and forth. This sudden movement can cause the brain to bounce around or twist in the skull, stretching and damaging the brain cells and creating chemical changes in the brain. (CDC, 2015)

- The most common form of a TBI is a mild TBI (MTBI), also known as a concussion, which accounts for $75 \%$ of those injured (Bergman \& Bay, 2010).

- Fall was the most commonly reported mechanism of injury. Followed by unintentional blunt trauma, motor vehicle collision, assault and sports related injuries (CDC, 2015).

- Although often considered mild, these injuries can lead to significant physical, cognitive, emotional and sleep impairments.

\section{Symptoms}

\section{Physical}

- Headaches, blurred vision, nausea or vomiting, dizziness, sensitivity to light or noise, balance problems \& fatigue.

\section{Cognitive}

- Difficulty thinking clearly, concentrating or remembering new information or feeling slowed down.

\section{Emotional/Mood}


- Irritability, depression, anxiety or heightened emotions.

\section{Sleep}

- Sleeping more or less than usual or difficulty falling asleep.

\section{Post-Concussion Syndrome (PCS)}

- A clinical state where three or more symptoms of concussion persist for more than three months after a head injury. (DSM-IV)

- Prolonged symptoms often lead to missed work and productivity as well as health care costs.

\section{Concussion Pathophysiology}

- A concussion is a transient neurological dysfunction that causes changes in the neurons referred to as the neurometabolic cascade. Immediately after injury to the brain there is a rapid release of neurotransmitters including glutamine and $\mathrm{N}$ methyl-D-aspartate which causes unchecked ionic fluxes of calcium and sodium entering the cell and potassium exiting the cell. In an attempt to restore ionic balance, the sodium potassium ATP pumps kick into high gear, triggering a dramatic jump in glucose metabolism (Giza \& Hoyda, 2001).

- There is a disparity between glucose supply and demand and in the setting of diminished blood flow leads to a cellular energy crisis (Giza \& Hoyda, 2001).

- Due to the inability to detect anatomical abnormalities on imaging and the ability of the deficits to resolve over time, some suggest that a concussion is a temporary neuronal dysfunction rather than cell death (Mapstone, 2016). 


\section{Prognosis}

- There is consistent evidence suggesting cognitive deficits in adults are present in the acute phase (48hrs to 2 weeks) and largely resolve within 3 months, but may last up to a year (Carroll et al., 2004).

- Mortality rates after concussion are considered rare.

- No studies found a connection between loss of consciousness (LOC) and increased cognitive deficits following a concussion (Carroll et a., 2014).

- LOC is NOT a requirement for concussion diagnosis.

- Most common complaints: fatigue (43\%), weakness (43\%), memory problems (40\%), headache (36\%) and dizziness (34\%) (Kraus, Schaffer, Ayers, Stenehjem, \& Shen, 2005).

- Common reasons for unplanned return visits to the emergency department (ED) include PCS (46\%), pain (18.7\%), and 14.6 were called back to ED for evaluation when test results came back, and $9.76 \%$ returned for evaluation after a repeat head injury (Ganti et al., 2015).

- Up to $1 / 3$ of patients will experience concussion related symptoms for up to a month after injury which can lead to anxiety (Haydel, 2012).

\section{Treatments}

- According to researchers, evidence is sufficient to recommend patient education including symptom-related information and supportive interaction (Comper, Bisschop, Carnide \& Tricco, 2005).

- Bed rest has NOT shown to improve outcomes. 
- The CDC HEADS UP Campaign recommends getting plenty of sleep at night, rest during the day and avoiding activities that are physically demanding or require a lot of concentration. They recommend gradually increasing activity but to stop and rest if symptomatic (CDC, 2015).

- In 2007 the CDC strongly emphasized the need for both physical and cognitive rest.

- Reassurance and support can alleviate anxiety.

\section{Discharge Education}

- Concussion patients discharged from the ED remember only $30 \%-50 \%$ of verbal instructions (Haydel, 2012).

- Verbal and written discharge instructions should be provided to all concussion patients and family members.

- Discharge instructions should include:

○ what to expect (signs \& symptoms)

- warning signs/red flags in which to return to the ED

instructions for returning to daily activities and work

follow up

- Most patients with concussion recover quickly and fully but some patients may take weeks, months or longer.

- The CDC HEADS UP Campaign has clearly written discharge instructions for patients. 
- With $80 \%$ of those with TBI treated and released directly for the ED, nurses are in a position to improve patient outcomes by providing evidence based discharge instructions to concussion patients (Bay \& Strong, 2011). 


\section{Appendix E}

Pre-Test Results by Question and Overall Score

\begin{tabular}{|c|c|c|c|c|c|c|c|c|c|c|c|}
\hline ID & Q 1 & $\mathrm{Q} 2$ & Q 3 & $\bar{Q} 4$ & Q 5 & Q 6 & Q 7 & Q 8 & Q 9 & Q10 & Score \\
\hline 1 & 10 & 10 & 0 & 0 & 10 & 10 & 10 & 0 & 0 & 10 & $60 \%$ \\
\hline 2 & 10 & 0 & 0 & 0 & 0 & 10 & 10 & 0 & 0 & 10 & $40 \%$ \\
\hline 3 & 10 & 10 & 10 & 10 & 10 & 0 & 10 & 0 & 0 & 0 & $60 \%$ \\
\hline 4 & 10 & 10 & 10 & 0 & 10 & 10 & 10 & 0 & 0 & 10 & $70 \%$ \\
\hline 5 & 10 & 10 & 0 & 10 & 10 & 10 & 10 & 0 & 10 & 0 & $70 \%$ \\
\hline 6 & 10 & 10 & 0 & 0 & 10 & 10 & 0 & 0 & 0 & 10 & $50 \%$ \\
\hline 7 & 10 & 10 & 0 & 10 & 0 & 10 & 10 & 0 & 0 & 0 & $50 \%$ \\
\hline 8 & 10 & 10 & 0 & 10 & 10 & 0 & 0 & 10 & 0 & 10 & $60 \%$ \\
\hline 9 & 10 & 10 & 0 & 10 & 10 & 10 & 10 & 10 & 0 & 10 & $80 \%$ \\
\hline 10 & 10 & 0 & 0 & 10 & 10 & 10 & 10 & 10 & 0 & 10 & $70 \%$ \\
\hline 11 & 10 & 10 & 10 & 10 & 10 & 10 & 10 & 10 & 0 & 10 & $90 \%$ \\
\hline 12 & 10 & 10 & 0 & 10 & 10 & 0 & 0 & 10 & 0 & 0 & $50 \%$ \\
\hline 13 & 10 & 10 & 0 & 10 & 0 & 0 & 10 & 0 & 0 & 10 & $50 \%$ \\
\hline 14 & 10 & 10 & 0 & 10 & 0 & 0 & 10 & 0 & 0 & 0 & $40 \%$ \\
\hline 15 & 10 & 10 & 0 & 10 & 10 & 10 & 10 & 10 & 10 & 10 & $90 \%$ \\
\hline 16 & 10 & 0 & 0 & 10 & 10 & 10 & 0 & 10 & 10 & 10 & $70 \%$ \\
\hline 17 & 0 & 10 & 0 & 10 & 0 & 0 & 10 & 10 & 0 & 0 & $40 \%$ \\
\hline 18 & 10 & 10 & 0 & 10 & 10 & 10 & 10 & 0 & 0 & 10 & $70 \%$ \\
\hline 19 & 10 & 10 & 0 & 10 & 10 & 10 & 10 & 0 & 0 & 10 & $70 \%$ \\
\hline 20 & 10 & 10 & 10 & 10 & 0 & 10 & 10 & 10 & 0 & 10 & $80 \%$ \\
\hline
\end{tabular}




\begin{tabular}{|c|c|c|c|c|c|c|c|c|c|c|c|}
\hline ID & Q 1 & Q 2 & Q 3 & Q 4 & Q 5 & Q 6 & Q 7 & Q 8 & Q 9 & Q10 & Score \\
\hline 21 & 10 & 0 & 10 & 0 & 10 & 10 & 10 & 10 & 0 & 0 & $60 \%$ \\
\hline 22 & 10 & 10 & 0 & 10 & 0 & 0 & 10 & 0 & 0 & 10 & $50 \%$ \\
\hline 23 & 0 & 10 & 0 & 10 & 10 & 0 & 10 & 0 & 0 & 10 & $50 \%$ \\
\hline 24 & 10 & 10 & 0 & 10 & 10 & 10 & 10 & 10 & 0 & 10 & $80 \%$ \\
\hline 25 & 10 & 10 & 0 & 0 & 10 & 0 & 0 & 10 & 0 & 0 & $40 \%$ \\
\hline 26 & 0 & 10 & 0 & 10 & 10 & 10 & 10 & 10 & 0 & 0 & $60 \%$ \\
\hline 27 & 10 & 10 & 0 & 10 & 10 & 10 & 0 & 10 & 0 & 10 & $70 \%$ \\
\hline 28 & 10 & 10 & 0 & 10 & 10 & 10 & 10 & 10 & 10 & 10 & $90 \%$ \\
\hline 29 & 10 & 10 & 10 & 0 & 10 & 10 & 10 & 0 & 0 & 0 & $60 \%$ \\
\hline 30 & 0 & 10 & 0 & 0 & 10 & 0 & 10 & 0 & 0 & 0 & $30 \%$ \\
\hline 31 & 0 & 10 & 0 & 10 & 10 & 0 & 10 & 0 & 0 & 10 & $50 \%$ \\
\hline 32 & 10 & 10 & 0 & 10 & 10 & 10 & 10 & 0 & 0 & 10 & $70 \%$ \\
\hline 33 & 10 & 0 & 0 & 10 & 10 & 0 & 10 & 10 & 10 & 0 & $60 \%$ \\
\hline 34 & 10 & 0 & 0 & 10 & 10 & 0 & 10 & 0 & 0 & 0 & $40 \%$ \\
\hline 35 & 10 & 0 & 0 & 10 & 10 & 10 & 10 & 10 & 0 & 0 & $60 \%$ \\
\hline 36 & 10 & 0 & 0 & 10 & 10 & 0 & 10 & 0 & 0 & 10 & $50 \%$ \\
\hline 37 & 10 & 10 & 0 & 0 & 10 & 0 & 0 & 0 & 0 & 10 & $40 \%$ \\
\hline 38 & 10 & 0 & 0 & 0 & 10 & 10 & 10 & 10 & 0 & 10 & $60 \%$ \\
\hline 39 & 10 & 10 & 10 & 10 & 10 & 10 & 0 & 0 & 10 & 10 & $80 \%$ \\
\hline 40 & 10 & 10 & 0 & 10 & 10 & 10 & 10 & 0 & 0 & 10 & $70 \%$ \\
\hline 41 & 10 & 10 & 10 & 10 & 10 & 0 & 10 & 10 & 0 & 0 & $70 \%$ \\
\hline
\end{tabular}




\begin{tabular}{|l|l|l|l|l|l|l|l|l|l|l|l|}
\hline ID & Q 1 & Q 2 & Q 3 & Q 4 & Q 5 & Q 6 & Q 7 & Q 8 & Q 9 & Q10 & Score \\
\hline 42 & 10 & 10 & 0 & 0 & 10 & 10 & 0 & 10 & 10 & 10 & $70 \%$ \\
\hline 43 & 10 & 10 & 0 & 10 & 10 & 0 & 0 & 0 & 0 & 0 & $40 \%$ \\
\hline 44 & 10 & 10 & 0 & 10 & 10 & 10 & 10 & 10 & 0 & 10 & $80 \%$ \\
\hline 45 & 10 & 10 & 0 & 10 & 10 & 10 & 10 & 10 & 0 & 10 & $80 \%$ \\
\hline 46 & 10 & 10 & 0 & 0 & 10 & 0 & 10 & 10 & 0 & 0 & $50 \%$ \\
\hline 47 & 10 & 10 & 0 & 0 & 10 & 10 & 10 & 10 & 0 & 10 & $70 \%$ \\
\hline 48 & 10 & 10 & 0 & 0 & 10 & 0 & 10 & 10 & 0 & 0 & $50 \%$ \\
\hline 49 & 10 & 10 & 10 & 10 & 10 & 10 & 10 & 10 & 0 & 10 & $90 \%$ \\
\hline 50 & 10 & 0 & 0 & 0 & 10 & 0 & 10 & 0 & 0 & 10 & $40 \%$ \\
\hline 51 & 10 & 10 & 10 & 10 & 0 & 10 & 10 & 10 & 0 & 10 & $80 \%$ \\
\hline
\end{tabular}




\section{Appendix F}

Post-Test Results by Question and Overall Score

\begin{tabular}{|c|c|c|c|c|c|c|c|c|c|c|c|}
\hline ID & Q1 & $\mathrm{Q} 2$ & Q3 & Q4 & Q5 & Q6 & Q7 & Q8 & Q9 & Q10 & Score \\
\hline 1 & 10 & 10 & 10 & 10 & 10 & 10 & 10 & 0 & 10 & 10 & $90 \%$ \\
\hline 2 & 10 & 10 & 10 & 10 & 10 & 10 & 10 & 10 & 0 & 10 & $90 \%$ \\
\hline 3 & 10 & 10 & 10 & 10 & 10 & 10 & 0 & 0 & 10 & 10 & $80 \%$ \\
\hline 4 & 10 & 10 & 10 & 10 & 10 & 10 & 0 & 10 & 0 & 10 & $80 \%$ \\
\hline 5 & 10 & 10 & 10 & 10 & 0 & 10 & 0 & 10 & 10 & 10 & $80 \%$ \\
\hline 6 & 10 & 10 & 0 & 10 & 10 & 10 & 10 & 10 & 0 & 10 & $80 \%$ \\
\hline 7 & 10 & 10 & 10 & 10 & 10 & 10 & 10 & 10 & 10 & 10 & $100 \%$ \\
\hline 8 & 10 & 10 & 10 & 10 & 10 & 10 & 10 & 10 & 0 & 10 & $90 \%$ \\
\hline 9 & 10 & 10 & 10 & 10 & 10 & 10 & 10 & 10 & 0 & 10 & $90 \%$ \\
\hline 10 & 10 & 10 & 10 & 10 & 0 & 10 & 0 & 0 & 0 & 10 & $60 \%$ \\
\hline 11 & 10 & 10 & 10 & 10 & 10 & 10 & 10 & 0 & 10 & 10 & $90 \%$ \\
\hline 12 & 10 & 0 & 10 & 10 & 10 & 0 & 10 & 0 & 0 & 10 & $60 \%$ \\
\hline 13 & 10 & 10 & 10 & 10 & 0 & 10 & 10 & 10 & 0 & 10 & $80 \%$ \\
\hline 14 & 10 & 10 & 10 & 0 & 10 & 10 & 10 & 0 & 0 & 10 & $70 \%$ \\
\hline 15 & 10 & 10 & 10 & 0 & 0 & 10 & 10 & 10 & 0 & 10 & $70 \%$ \\
\hline 16 & 10 & 10 & 10 & 10 & 10 & 10 & 10 & 0 & 10 & 10 & $90 \%$ \\
\hline 17 & 10 & 10 & 0 & 10 & 0 & 0 & 10 & 0 & 0 & 10 & $50 \%$ \\
\hline 18 & 10 & 10 & 10 & 10 & 10 & 10 & 10 & 10 & 10 & 10 & $100 \%$ \\
\hline 19 & 10 & 10 & 10 & 10 & 0 & 10 & 10 & 10 & 10 & 10 & $90 \%$ \\
\hline 20 & 10 & 10 & 0 & 10 & 0 & 0 & 10 & 0 & 0 & 0 & $40 \%$ \\
\hline
\end{tabular}




\begin{tabular}{|l|l|l|l|l|l|l|l|l|l|l|l|}
\hline ID & Q1 & Q2 & Q3 & Q4 & Q5 & Q6 & Q7 & Q8 & Q9 & Q10 & Score \\
\hline 21 & 10 & 10 & 10 & 10 & 10 & 10 & 10 & 10 & 10 & 10 & $100 \%$ \\
\hline 22 & 10 & 10 & 10 & 10 & 10 & 10 & 0 & 0 & 10 & 10 & $80 \%$ \\
\hline 23 & 10 & 10 & 10 & 10 & 10 & 10 & 0 & 0 & 0 & 0 & $60 \%$ \\
\hline 24 & 10 & 10 & 10 & 10 & 0 & 10 & 0 & 0 & 10 & 10 & $70 \%$ \\
\hline 25 & 10 & 10 & 10 & 10 & 10 & 10 & 0 & 0 & 0 & 10 & $70 \%$ \\
\hline 26 & 10 & 10 & 10 & 0 & 10 & 10 & 10 & 10 & 0 & 10 & $80 \%$ \\
\hline 27 & 10 & 10 & 10 & 10 & 10 & 10 & 0 & 0 & 10 & 10 & $80 \%$ \\
\hline 28 & 10 & 10 & 10 & 10 & 0 & 10 & 0 & 10 & 0 & 10 & $70 \%$ \\
\hline
\end{tabular}

